\title{
Dissecting the mechanism of carotid atherosclerosis from the perspective of regulation
}

\author{
$\mathrm{MIN} \mathrm{LIN}^{1 *}, \mathrm{LIN}_{\mathrm{ZHAO}}{ }^{*}, \mathrm{WENLONG} \mathrm{ZHAO}^{1}$ and $\mathrm{JING}_{\mathrm{WENG}}{ }^{1}$ \\ Departments of ${ }^{1}$ Neurology, and ${ }^{2}$ Neurosurgery, Fuzhou General Hospital of Nanjing Command, PLA, Fuzhou 350025, P.R. China
}

Received February 20, 2014; Accepted August 28, 2014

DOI: $10.3892 /$ ijmm.2014.1960

\begin{abstract}
Carotid atherosclerosis is a chronic inflammatory disease of the arterial wall. The present study aimed to identify changes in the gene expression and regulatory factors for atherosclerotic plaques of carotid atherosclerosis from an early to an advanced stage. The original data were downloaded from the NCBI GEO database under accession no. GSE28829. Differentially expressed genes (DEGs) were detected by the Robust Multiarray Average (RMA). The enriched Gene Ontology (GO) terms and pathways for DEGs using DAVID were subsequently identified. The transcriptional and microRNA (miRNA) regulatory network were constructed for the DEGs. Cis-regulatory signals were also investigated. More genes were activated in the advanced stage compared with the early stage. IGHG1 and SPP1 were upregulated, while MYBL1 and PLD were downregulated. The upregulated genes in the advanced stage were involved in atherosclerosis-related GO terms such as immune, vascular and cell movement homeostasis. The DEGs were significantly enriched in cell adhesion molecules (CAMs) and the focal adhesion pathway. MMP9 and CFL2 played key roles in the transcriptional regulatory network. Moreover, miR-328 was identified as one of the hubs in the miRNA regulatory network. The results may therefore be used to determine the mechanism involved in carotid atherosclerosis.
\end{abstract}

\section{Introduction}

Atherosclerosis is a chronic disease that remains asymptomatic for decades (1). It is caused by the formation of multiple plaques within the arteries. Attention has been focused on the 'vulnerable plaque' since the late 1990s onwards (2).

Correspondence to: Dr Min Lin, Department of Neurology, Fuzhou General Hospital of Nanjing Command, PLA, 256 Xierhuan Road, Fuzhou, Fujian 350025, P.R. China

E-mail: minlin232@hotmail.com

*Contributed equally

Key words: carotid atherosclerosis, differentially expressed genes, transcription factors, miRNA
Atherosclerosis can lead to ischemic heart disease, cerebrovascular accidents and peripheral vascular diseases (3). Carotid intima-media thickness (cIMT) level is known as a surrogate marker of atherosclerosis (4). A special type of carotid atherosclerosis with CagA-positive Helicobacter pylori $\left(\mathrm{CagA}^{+} \mathrm{HP}\right)$ infection is common in China. An increased serum YKL-40 level suggests plaque instability and more severe clinical symptoms of carotid atherosclerosis with $\mathrm{CagA}^{+} \mathrm{HP}$ infection (5). Inflammatory cytokines induced by VEGF, such as monocyte chemoattractant protein (MCP-1), have been previously shown to be involved in the pathogenesis and progression of carotid atherosclerosis (6).

Metabolic syndrome may be independently associated with the early stage but not the later and advanced stages of carotid atherosclerosis in community residents in China (7). An in vivo 3T MRI study was previously conducted to determine the effect of gender differences of high-risk carotid atherosclerotic plaque with $<50 \%$ stenosis in asymptomatic patients (8). Evaluation of carotid atherosclerosis was therefore performed from the perspective of blood flow reflection (9). Results of a multivariate analysis revealed plaque number by ultrasonography $(\mathrm{P}=0.023)$, age $(\mathrm{P}=0.001)$, calcium-phosphate product $(\mathrm{P}=0.049)$ and serum albumin $(\mathrm{P}=0.009)$ as independent risk factors (10). Higher brachial-ankle pulse wave velocity was identified as a risk factor for carotid atherosclerosis in patients with end-stage renal disease (11). The relationship between levels of circulating intercellular cell-adhesion molecule-1 (cICAM-1) or P-selectin (cP-selectin) and the severity of carotid atherosclerosis was also examined (12). The findings of that study showed that $\mathrm{cP}$-selectin did not increase until atherosclerosis was at an advanced stage (12).

Inactivation of the PDZK1 gene is known to promote the development of aortic root atherosclerosis in apolipoprotein $\mathrm{E}$ (apoE) KO mice fed with a high fat/high cholesterol diet (13). Additionally, SERPINA1 was found to be upregulated in atherosclerotic plaques (14). Angiogenesis, the process of new capillary formation from existing blood vessels, is dysregulated in many pathological disorders including atherosclerosis (15). In $\mathrm{CD}^{+} 8^{+}$cells from regressing plaque of atherosclerosis, Feig et al (16) observed that genes related to cell adhesion, such as cadherins and vinculin, were downregulated.

Transcription factors such as LDLR and TP53 are important in the development of atherosclerosis, as identified in a Malaysian study population (13). It has been previously reported that miRNAs are associated with atherosclerosis (17). 
However, the association between miRNAs and atherosclerosis has not yet been fully elucidated.

Immune-associated biological processes can affect atherosclerosis. Growth differentiation factor-15 deficiency inhibits the progression of atherosclerosis by controlling the interleukin-6-dependent inflammatory response to vascular injury (18). Vascular smooth muscle cell (VSMC) phenotypic modulation plays a key role in atherosclerosis. SMCs secrete cytokines and express cell adhesion molecules such as IL-8, IL-6 and VCAM-1 (19). Prenatal arsenic exposure alters gene expression in the adult liver to a proinflammatory state contributing to accelerated atherosclerosis by affecting pathways such as antigen processing and presentation (20). Vascular smooth muscle contraction is a main effect for artery (21). Morelloflavone, a biflavonoid and an active ingredient of the plant, has been shown to inhibit VSMC migration through its inhibition of multiple migration-related kinases such as focal adhesion kinase (22).

Therefore, in the present study, we initially detected differentially expressed genes (DEGs). Subsequently, we identified the enriched Gene Ontology (GO) terms and pathways for the DEGs. The transcriptional and miRNA regulatory network for the DEGs was also constructed. Cis-regulatory signals were also investigated.

\section{Materials and methods}

Data preprocessing. The original data were downloaded from the National Center for Biotechnology Gene Expression Omnibus (NCBI GEO) database for atherosclerotic plaques of carotid atherosclerosis under accession no. GSE28829 (23), including 13 chips at early stage (EAR, pathological, intimal thickening and intimal xanthoma) and 16 chips at advanced stage (ADV, thin or thick fibrous cap atheroma). The chip platform was GPL570, Affymetrix Human Genome U133 Plus 2.0 Array.

Background subtraction and quantile normalization was performed using Affymetrix Power Tools (APT) (http:// www.affymetrix.com/) in the Robust Multiarray Average (RMA) algorithm (24). Genes with a low expression were filtered, ensuring that their plier was $\geq 100$ in at least $2 / 3$ of the samples (25).

Identifying differentially expressed genes. Criteria for a differential probe included: i) signal strength of one probe is $>1.5$-fold between the two groups; ii) t-test P-value of $\leq 0.01$; and iii) plier $\geq 100$ in at least $2 / 3$ of the samples.

Functional enrichment of differential genes. Gene Ontology (GO) and the Kyoto Encyclopedia of Genes and Genomes (KEGG) pathway enrichment analysis was conducted with DAVID (26) for the differential genes between two groups. The threshold [P-value $\leq 0.05$ and false discovery rate (FDR) $\leq 0.05]$ was calculated using Fisher's Exact test.

Transcriptional regulatory network. The differential genes, especially atherosclerosis-related genes, may be regulated by different transcriptional factors and miRNAs in different stages. We initally extracted the sequences located at $1 \mathrm{k} \mathrm{bp}$ upstream and $200 \mathrm{bp}$ downstream of 5'UTR for the transcrip-

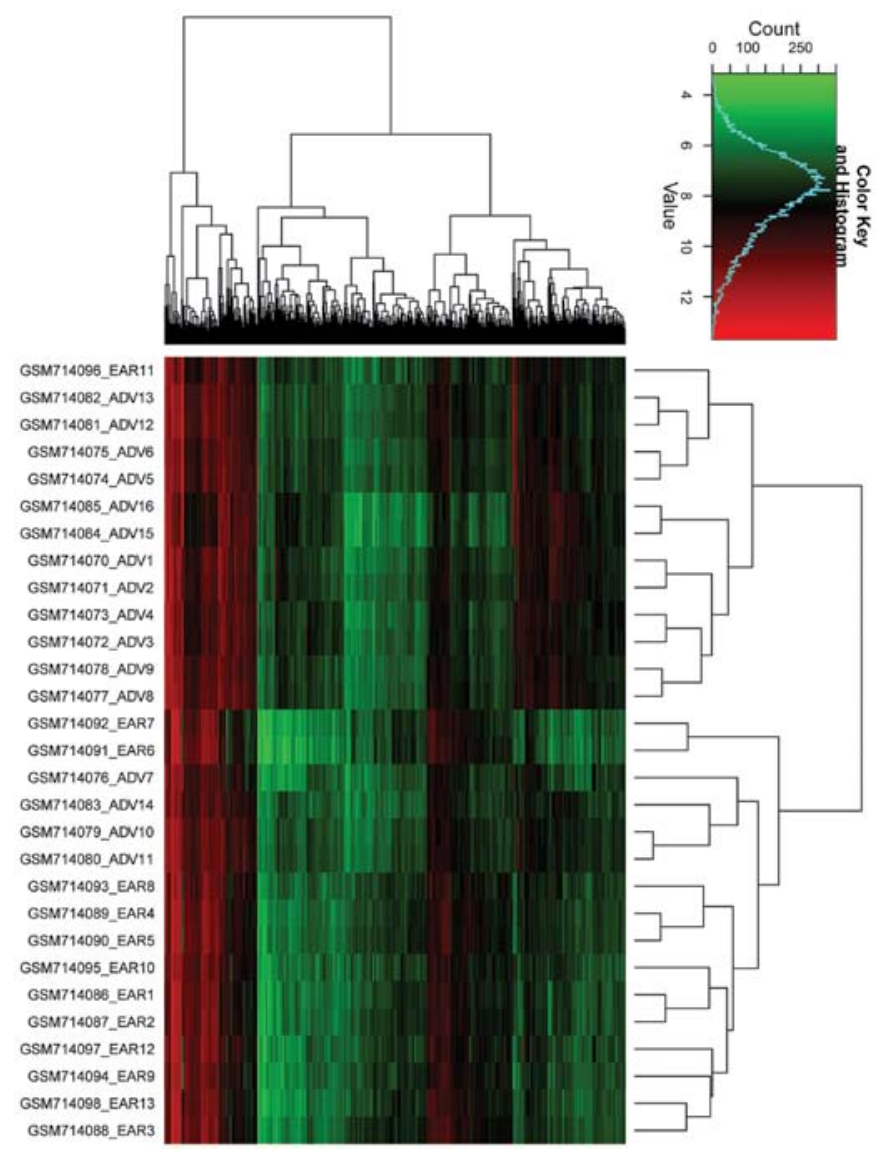

Figure 1. Hierarchical clustering analysis of 889 differentially expressed transcripts in all samples.

tion start site, using JASPAR (27) (http://jaspar.genereg.net) (the score threshold was 0.95 ). The core transcriptional regulatory network was constructed for the upregulated genes, with a $>4$-fold change in the advanced stage and a $>2$-fold change in the early stage. Upregulated genes were regulated by transcriptional factors (TFs) in the early and advanced stages of carotid atherosclerosis. TFs act depending on cis-regulatory motif in 5'UTR of their target genes. In order to detect such significant enriched cis-regulatory signals, hypergeometric distribution test was implemented (28), with a P-value of $\leq 0.05$.

miRNA regulatory network . The $1 \mathrm{k}$ bp 3'UTR sequence was extracted from the differentially expressed genes. Although the binding of miRNA and its targets in animals are not as conservative as in plant, while binding is relatively conservative in the seed region. RNAhybrid is based conservation in seed region, which makes it more suitable for animal miRNA target gene prediction. RNAhybrid (29) was subsequently used to identify miRNA binding sites (P-value $\leq 0.01$ ).

\section{Results}

Differentially expressed genes. In the present study, the early stage sample was considered as the control group. We identified 889 differential probes (corresponding to 707 genes), of which 413 probes (322 genes) were downregulated, and 


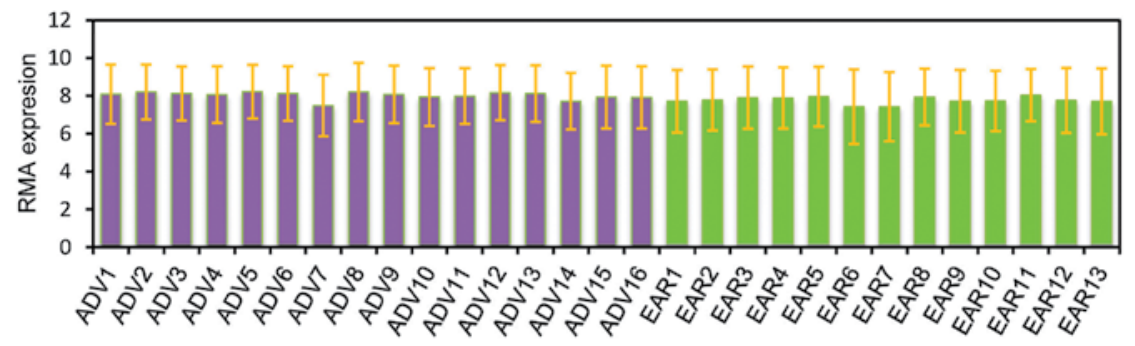

Figure 2. The average expression value of 889 differentially expressed transcripts in each sample. Purple bars are the advanced samples and green bars are the early stage sample. Bar values are the standard error of the mean (SEM).
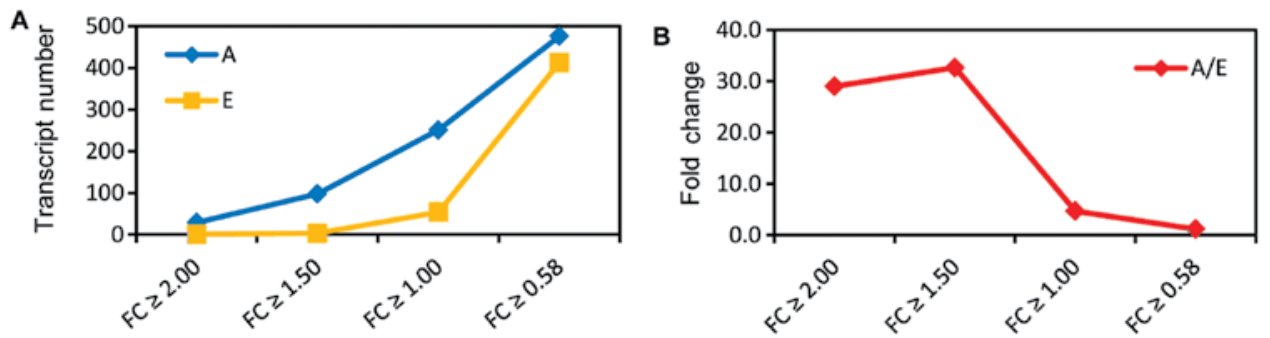

Figure 3. The distribution of 889 differentially expressed transcripts (DETs) in the early and advanced stages. (A) The number of transcripts with a different FC. Blue shows the advanced stage samples, while yellow shows the early stage samples. (B) The fold change of DETs in the advanced samples when compared with its expression in the early stage samples with a different FC. FC represents the $\log 2$ fold change.

Table I. Major biological processes enriched by upregulated genes in early stage.

\begin{tabular}{lccr}
\hline Term & Count & P-value & FDR \\
\hline GO:0007010: Cytoskeleton organization & 25 & $2.12 \mathrm{E}-07$ & $3.51 \mathrm{E}-04$ \\
GO:0007155: Cell adhesion & 29 & $1.14 \mathrm{E}-05$ & $1.88 \mathrm{E}-02$ \\
GO:0007517: Muscle organ development & 14 & $4.57 \mathrm{E}-05$ & $7.56 \mathrm{E}-02$ \\
GO:0006937: Regulation of muscle contraction & 7 & $1.16 \mathrm{E}-03$ & $1.90 \mathrm{E}+00$ \\
GO:0001558: Regulation of cell growth & 11 & $1.38 \mathrm{E}-03$ & $2.25 \mathrm{E}+00$ \\
GO:0044057: Regulation of system process & 14 & $1.80 \mathrm{E}-03$ & $2.93 \mathrm{E}+00$ \\
GO:0010959: Regulation of metal ion transport & 7 & $2.13 \mathrm{E}-03$ & $3.46 \mathrm{E}+00$ \\
GO:0007507: Heart development & 11 & $2.92 \mathrm{E}-03$ & $4.73 \mathrm{E}+00$ \\
\hline
\end{tabular}

$\mathrm{P} \leq 0.05$ and false discovery rate $(\mathrm{FDR}) \leq 0.05$.

476 probes (385 genes) were upregulated in advanced stage samples.

The 889 differential probes were clustered using $\mathrm{R}$ hclust based on RMA $\log 2$-transformed values (Fig. 1). Thirteen samples were clustered into one group, with only one early stage sample being mis-clustered in this group. The standard error of the mean distribution is shown in Fig. 2. Fig. 3 shows the up- and downregulation for the 889 differential probes (corresponding to 708 genes). As the absolute log fold change $(\log \mathrm{FCl})$ becomes larger, the number of upregulated genes were reduced (Fig. 3A). However, the fold change increased as the $\log \mathrm{FCl}$ became larger (Fig. 3B). For example, when $\mid \log \mathrm{FCl} \geq 1.5$, the fold change was 0.58 , whereas when $\mid \log F C l \geq 1$, the fold change was 1 . This finding suggested that a large number of genes were activated in the advanced stage.
Enriched functional terms of differential genes. The downregulated genes were enriched in biological processes including cytoskeleton organization, cell adhesion, muscle organ development, regulation of muscle contraction, regulation of cell growth, regulation of system process, regulation of metal ion transport, heart development, muscle contraction, muscle cell differentiation, negative regulation of cell growth, intracellular signaling cascade, myofibril assembly, cellular metal ion homeostasis, regulation of calcium ion transport, negative regulation of cell size and regulation of intracellular transport (Table I). The findings demonstrated that the biological processes do not correlate with atherosclerosis.

The upregulated genes enriched in biological processes associated with atherosclerosis were: i) immune-associated GO terms, including defense response, response to wounding, 
Table II. Major biological processes enriched by upregulated genes in advanced stage.

\begin{tabular}{|c|c|c|c|}
\hline Term & Count & P-value & FDR \\
\hline GO:0006955: Immune response & 86 & $4.85 \mathrm{E}-40$ & $8.42 \mathrm{E}-37$ \\
\hline GO:0006952: Defense response & 65 & $1.40 \mathrm{E}-25$ & $2.43 \mathrm{E}-22$ \\
\hline GO:0009611: Response to wounding & 57 & $1.10 \mathrm{E}-22$ & $1.91 \mathrm{E}-19$ \\
\hline GO:0006954: Inflammatory response & 43 & 2.13E-20 & $3.69 \mathrm{E}-17$ \\
\hline GO:0006935: Chemotaxis & 27 & $2.20 \mathrm{E}-15$ & $3.85 \mathrm{E}-12$ \\
\hline GO:0042330: Taxis & 27 & $2.20 \mathrm{E}-15$ & $3.85 \mathrm{E}-12$ \\
\hline GO:0002252: Immune effector process & 21 & $2.08 \mathrm{E}-11$ & $3.61 \mathrm{E}-08$ \\
\hline GO:0048584: Positive regulation of response to stimulus & 27 & $2.37 \mathrm{E}-11$ & 4.11E-08 \\
\hline GO:0007626: Locomotory behavior & 29 & $2.40 \mathrm{E}-11$ & 4.16E-08 \\
\hline GO:0002443: Leukocyte mediated immunity & 17 & $7.37 \mathrm{E}-11$ & $1.28 \mathrm{E}-07$ \\
\hline $\begin{array}{l}\text { GO:0002504: Antigen processing and presentation of peptide } \\
\text { or polysaccharide antigen via MHC class II }\end{array}$ & 12 & $8.24 \mathrm{E}-11$ & $1.43 \mathrm{E}-07$ \\
\hline GO:0002250: Adaptive immune response & 16 & $1.47 \mathrm{E}-10$ & $2.55 \mathrm{E}-07$ \\
\hline GO:0002253: Activation of immune response & 17 & $2.97 \mathrm{E}-10$ & $5.16 \mathrm{E}-07$ \\
\hline GO:0002449: Lymphocyte mediated immunity & 15 & $4.22 \mathrm{E}-10$ & 7.33E-07 \\
\hline GO:0019882: Antigen processing and presentation & 16 & $4.49 \mathrm{E}-10$ & 7.79E-07 \\
\hline GO:0007610: Behavior & 33 & $2.54 \mathrm{E}-08$ & $4.41 \mathrm{E}-05$ \\
\hline GO:0019724: B cell-mediated immunity & 12 & 3.99E-08 & $6.92 \mathrm{E}-05$ \\
\hline GO:0002478: Antigen processing and presentation of exogenous peptide antigen & 7 & $5.36 \mathrm{E}-08$ & $9.29 \mathrm{E}-05$ \\
\hline GO:0007155: Cell adhesion & 40 & $1.72 \mathrm{E}-07$ & $2.98 \mathrm{E}-04$ \\
\hline GO:0001568: Blood vessel development & 22 & $1.75 \mathrm{E}-07$ & $3.04 \mathrm{E}-04$ \\
\hline GO:0002526: Acute inflammatory response & 14 & $3.02 \mathrm{E}-07$ & $5.23 \mathrm{E}-04$ \\
\hline GO:0019884: Antigen processing and presentation of exogenous antigen & 7 & 3.29E-07 & $5.70 \mathrm{E}-04$ \\
\hline GO:0001775: Cell activation & 23 & $6.13 \mathrm{E}-07$ & $1.06 \mathrm{E}-03$ \\
\hline GO:0030334: Regulation of cell migration & 17 & $1.43 \mathrm{E}-06$ & $2.48 \mathrm{E}-03$ \\
\hline GO:0048514: Blood vessel morphogenesis & 19 & $1.45 \mathrm{E}-06$ & $2.51 \mathrm{E}-03$ \\
\hline GO:0051270: Regulation of cell motion & 18 & $1.83 \mathrm{E}-06$ & $3.18 \mathrm{E}-03$ \\
\hline GO:0055066: Di-, tri-valent inorganic cation homeostasis & 20 & $2.13 \mathrm{E}-06$ & $3.69 \mathrm{E}-03$ \\
\hline GO:0045321: Leukocyte activation & 20 & $2.56 \mathrm{E}-06$ & $4.44 \mathrm{E}-03$ \\
\hline GO:0006956: Complement activation & 9 & $3.78 \mathrm{E}-06$ & $6.56 \mathrm{E}-03$ \\
\hline GO:0002541: Activation of plasma proteins involved in acute inflammatory response & 9 & 4.55E-06 & $7.90 \mathrm{E}-03$ \\
\hline GO:0001525: Angiogenesis & 15 & $6.60 \mathrm{E}-06$ & $1.14 \mathrm{E}-02$ \\
\hline GO:0042592: Homeostatic process & 38 & $6.64 \mathrm{E}-06$ & $1.15 \mathrm{E}-02$ \\
\hline GO:0040012: Regulation of locomotion & 17 & $7.55 \mathrm{E}-06$ & $1.31 \mathrm{E}-02$ \\
\hline GO:0009617: Response to bacterium & 17 & 8.07E-06 & $1.40 \mathrm{E}-02$ \\
\hline GO:0055080: Cation homeostasis & 21 & 8.17E-06 & $1.42 \mathrm{E}-02$ \\
\hline GO:0050865: Regulation of cell activation & 16 & $1.04 \mathrm{E}-05$ & $1.80 \mathrm{E}-02$ \\
\hline GO:0048878: Chemical homeostasis & 29 & $1.48 \mathrm{E}-05$ & $2.57 \mathrm{E}-02$ \\
\hline GO:0006874: Cellular calcium ion homeostasis & 16 & $1.77 \mathrm{E}-05$ & $3.07 \mathrm{E}-02$ \\
\hline
\end{tabular}

$\mathrm{P} \leq 0.05$ and false discovery rate $(\mathrm{FDR}) \leq 0.05$.

inflammatory response, immune effector process, leukocyte mediated immunity, antigen processing and presentation of peptide or polysaccharide antigen via the MHC class II, adaptive immune response, activation of immune response, lymphocyte mediated immunity, antigen processing and presentation, behavior, B cell-mediated immunity, antigen processing and presentation of exogenous peptide antigen, acute inflammatory response, chemotaxis, antigen processing and presentation of exogenous antigen; ii) vascular-related GO terms including blood vessel development, blood vessel 
Table III. Enriched pathways of differentially expressed transcripts.

\begin{tabular}{|c|c|c|c|}
\hline Term & Count & P-value & FDR \\
\hline hsa05322: Systemic lupus erythematosus & 21 & $3.65 \mathrm{E}-08$ & 0.00004 \\
\hline hsa04514: Cell adhesion molecules (CAMs) & 23 & $2.78 \mathrm{E}-07$ & 0.00033 \\
\hline hsa04612: Antigen processing and presentation & 18 & $3.26 \mathrm{E}-07$ & 0.00039 \\
\hline hsa04610: Complement and coagulation cascades & 16 & $7.32 \mathrm{E}-07$ & 0.00087 \\
\hline hsa05310: Asthma & 10 & $5.73 \mathrm{E}-06$ & 0.00681 \\
\hline hsa05416: Viral myocarditis & 15 & $5.92 \mathrm{E}-06$ & 0.00704 \\
\hline hsa04142: Lysosome & 19 & $1.13 \mathrm{E}-05$ & 0.01347 \\
\hline hsa04672: Intestinal immune network for IgA production & 12 & $1.66 \mathrm{E}-05$ & 0.01978 \\
\hline
\end{tabular}

P-value $\leq 0.05$ and false discovery rate $(\mathrm{FDR}) \leq 0.05$.

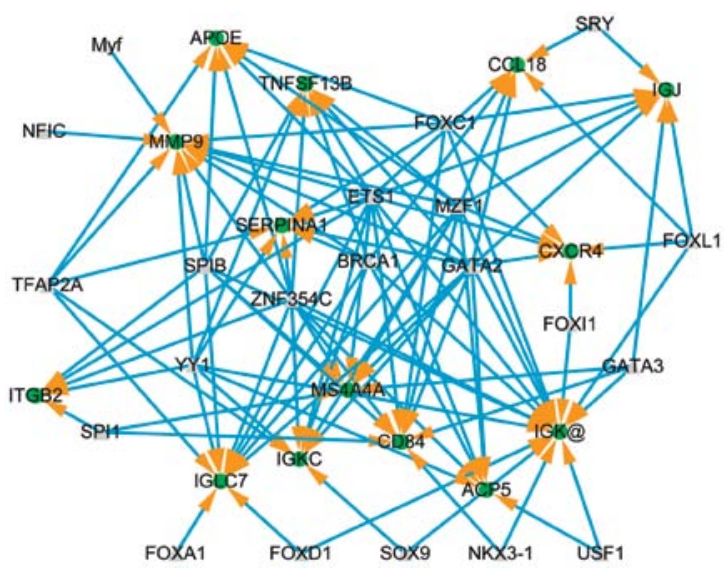

Figure 4. The core transcriptional regulatory network of upregulated genes (RMA $\log 2$ transformed values $>2$ ). Green nodes are the target genes, and the other nodes are the transcription factors. Arrows show that transcription factors target the genes.

morphogenesis, and angiogenesis; and iii) cell movement homeostasis including cell adhesion, regulation of cell motion, regulation of cell migration, regulation of locomotion, cation homeostasis and chemical homeostasis (Table II).

The enriched pathways of differential genes included systemic lupus erythematosus, antigen processing and presentation, complement and coagulation cascades, asthma, viral myocarditis, lysosome, intestinal immune network for IgA production (Table III).

Transcriptional regulatory network. The core transcriptional regulatory network is shown in Fig. 4 for upregulated genes (RMA $\log 2$ transformed values $>2$ ). Fourteen upregulated genes are shown, targeted by 21 transcription factors, constructing 111 regulatory relationships. The hub genes are IGK (15 neighbors), MMP9 (11 neighbors) and IGLC7 (10 neighbors).

The core transcriptional regulatory network is shown in Fig. 5 for downregulated genes (RMA $\log 2$ transformed values <-1). Forty-five downregulated genes, targeted by 22 transcription factors, constructing 324 regulatory relationships. The hub genes are AW451999 (10 neighbors), CFL2 (10 neighbors) and PDZRN3 (10 neighbors).

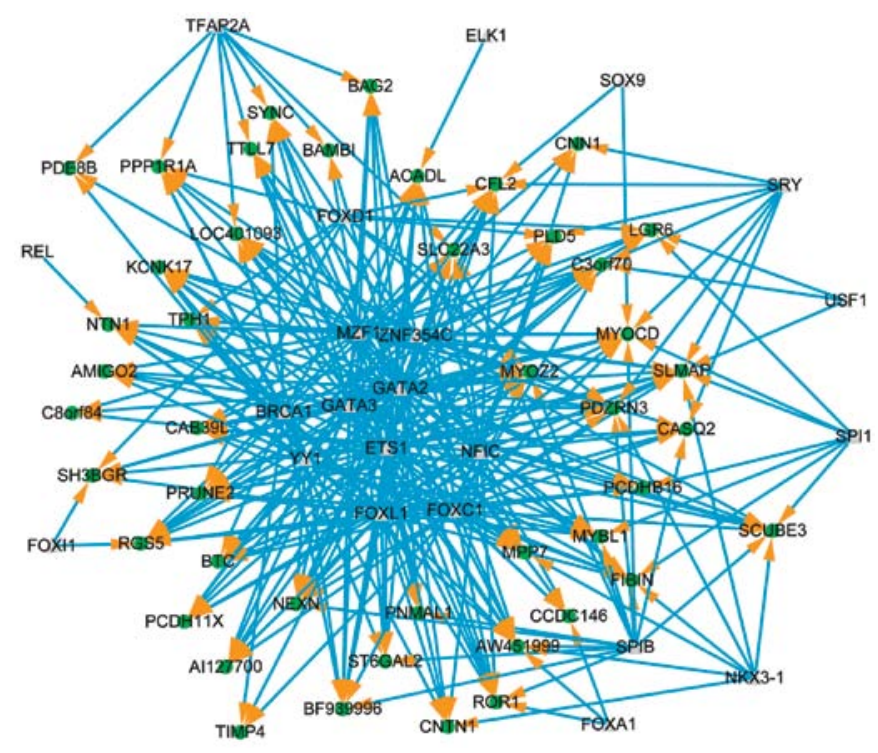

Figure 5. The core transcriptional regulatory network of downregulated genes (RMA $\log 2$ transformed values <-1). Green nodes are the target genes, and the other nodes are the transcription factors. Arrows show that transcription factors target the genes.

Significantly enriched cis-regulatory signals. Fig. 6A shows the 5'UTR of upregulated genes enriched in TF motif Myf (P-value 1.33E-02) and MZF1 (P-value 2.47E-02). By contrast, Fig. 6B shows the 5'UTR of downregulated genes enriched in TF motif FOXL1 (P-value, 5.95E-03) and NKX3-1 (P-value, 3.57E-02).

miRNA regulatory network. The core miRNA regulatory network is shown in Fig. 7 for the upregulated genes (RMA $\log 2$ transformed values $>2$ ). The genes identified by arrows are 13 upregulated genes, targeted by 262 miRNAs, comprising 372 regulatory relationships. The hub genes are IGLC7 (78 miRNAs target it), APOE (66 miRNAs) and SERPINA1 (53 miRNAs). The hub miRNAs are miR-6756 (8 target genes), miR-328 (6 genes) and miR-6803 (5 genes).

The core miRNA regulatory network is shown in Fig. 8 for the downregulated genes (RMA $\log 2$ transformed values $>1$ ). The genes identified by arrows are 33 downregulated genes, targeted by 295 miRNAs, comprising 561 regulatory relation- 
A

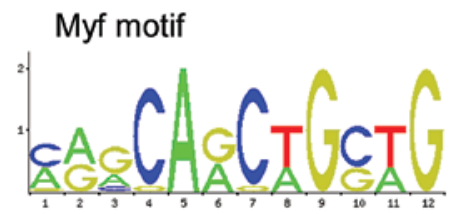

B

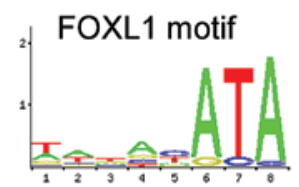

MZF1 motif
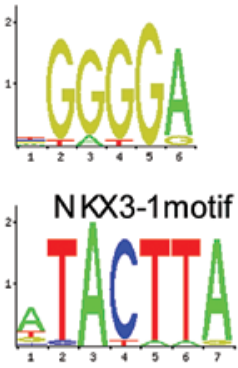

Figure 6. Significantly enriched TF motifs by DETs. Enriched motifs of (A) upregulated and (B) downregulated genes.

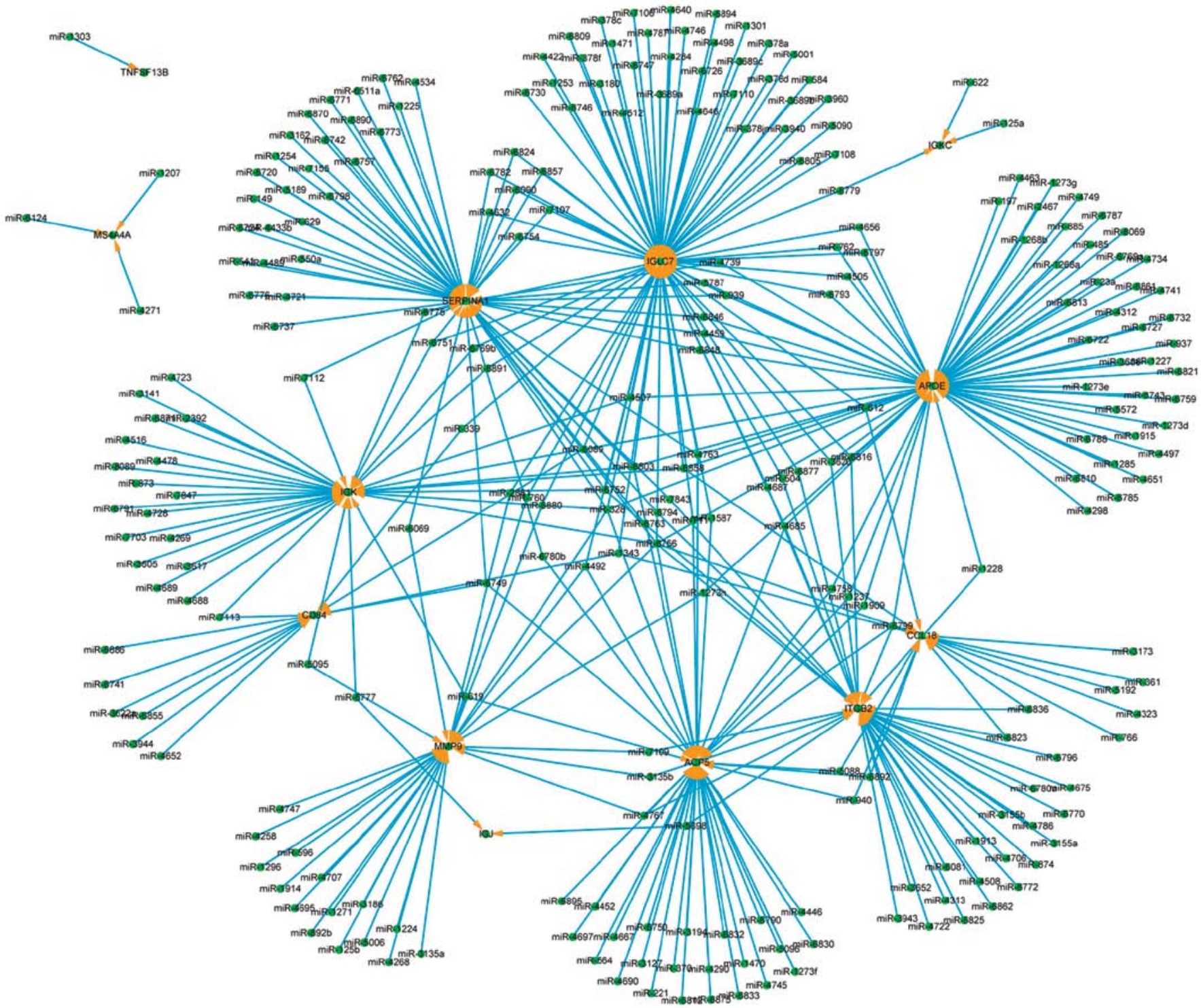

Figure 7. The core miRNA regulatory network of upregulated genes (RMA $\log 2$ transformed values $>2$ ) in advanced stage samples.

ships. The hub genes are LGR6 (71 miRNAs target it), NTN1 (61 miRNAs), CNN1 (59 miRNAs) and PDZRN3 (57 miRNAs). The hub miRNAs are miR-6756 (11 target genes), miR-619 (10 genes), miR-6089 (8 genes) and miR-6803 (8 genes).

\section{Discussion}

Carotid atherosclerosis is defined as the presence of atherosclerotic plaques in any of the carotid vessel segments (30). In the 


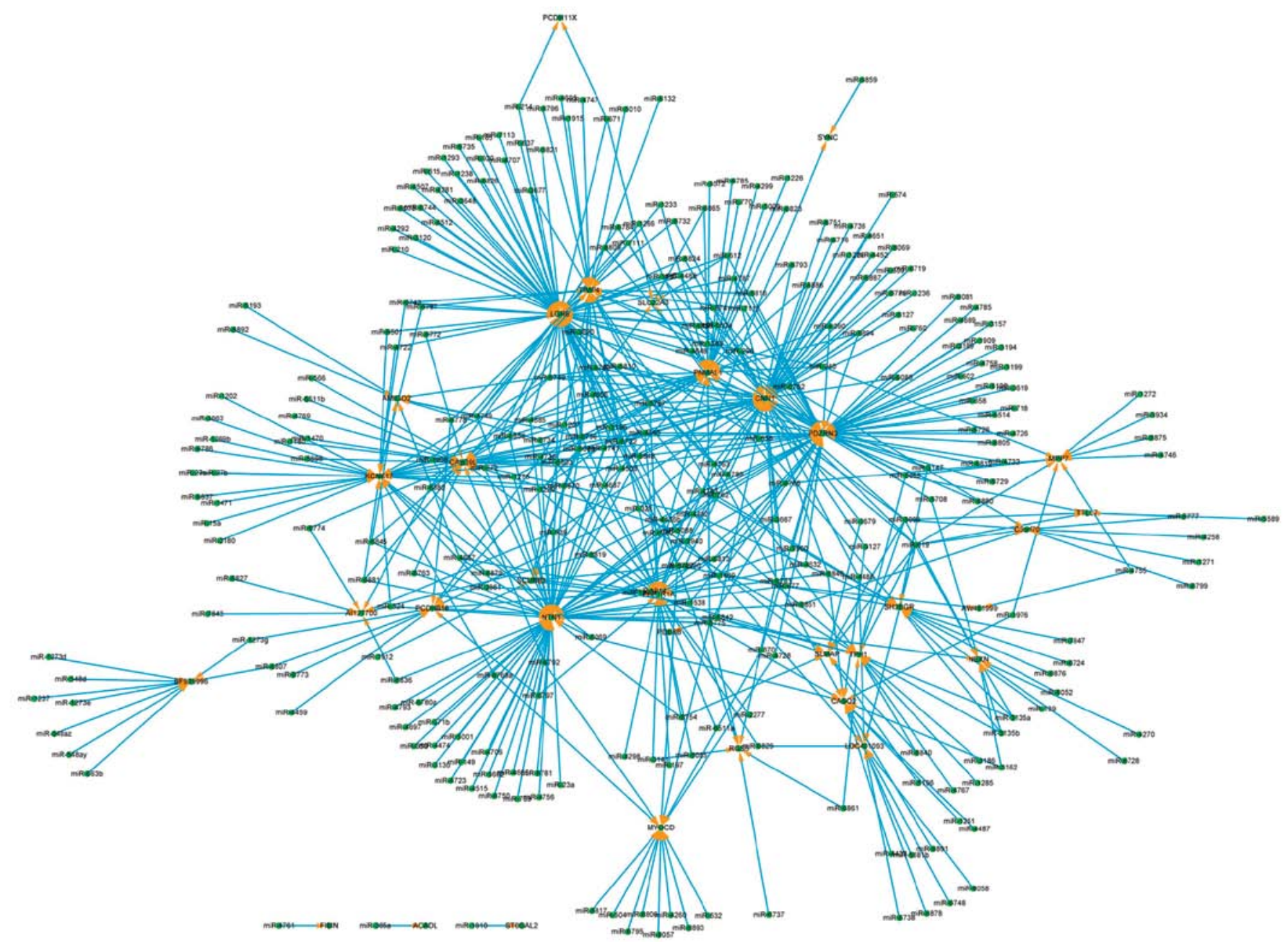

Figure 8 . The core miRNA regulatory network of upregulated genes (RMA $\log 2$ transformed values $>2$ ) in the early stage samples.

present study, we aimed to identify gene expression changes and regulatory factors for carotid atherosclerosis from an early to an advanced stage. More genes were activated in advanced stage compared with early stage. The upregulated genes in the advanced stage were involved in GO terms including immune, vascular and cell movement homeostasis. The differentially expressed genes (DEGs) were significantly enriched in cell adhesion molecules (CAMs) and focal adhesion. Genes such as MMP9 and CFL2 played key roles in the transcriptional regulatory network. Moreover, miR-328 was one of the hubs in the miRNA regulatory network.

A total of 889 transcripts were identified to be differentially expressed from early stage plaques of carotid atherosclerosis to advanced stage plaques. As shown in Fig. 3B, A/E increased when the fold change threshold was elevated. The majority of the DEGs were upregulated in the advanced stage, while they were inhibited in the early stage.

The DEGs activated in the advanced stage may correlate with plaques of carotid atherosclerosis and various types of cancer. A number of immune system-related cells were detected in human carotid atherosclerosis patients such as monocytes/macrophages, $\mathrm{T}$ cells and plasmacytoid dendritic cells (pDCs) (23). IGHG1 (Ig $\gamma-1$ chain $\mathrm{C}$ region) is one gene associated with 'innate immune response'. IGHG1 expression has been reported to correlate with immune evasion mechanisms, which contribute to the proliferation of human pancreatic cancer (31). Furthermore, inhibiting IGHG1 expression by siRNA leads to cancer growth inhibition and apoptosis in prostate cancer (32). SPP1 (osteopontin) is involved as a cytokine in type I immunity to elevate the product of interferon- $\gamma$ and interleukin-12. On the other hand, SPP1 reduces the expression of interleukin-10, and IFN- $\gamma$ treatment, resulting in an increase of cytokines/cytokine receptors including CSF2, IL1R2 and SPP1 (33). In addition, smoking can increase SPP1 expression, and subsequently induce inflammation and emphysema (34). IGKC (Ig $\kappa$ chain C region) participates in humoral immune response, which has been reported to play key roles in non-small cell lung cancer and breast cancer (35-37). The genes activated in early stage do not possess a similar function, including BTC, MYBL1 and PLD.

Immune-associated GO terms, vascular-related GO terms and cell movement homeostasis were identified to be associated with atherosclerosis. The IL-6-gp130 axis is a key regulator of inflammatory acute phase signaling in hepatocytes for the development of atherosclerosis (38). Blood flow is crucial for blood vessel development during embryogenesis and for the regulation of vessel diameter in adult life. It is also a key factor in atherosclerosis, which occurs mainly in regions of arteries that experience disturbances in fluid flow (39). Fibrinolytic balance and the potential contribution of PAI-1 to the regula- 
tion of cell migration are involved in the pathogenesis of the simple atherosclerotic lesions observed in the mouse (40).

Leukocyte transendothelial migration is one of the earliest events of immune inflammatory responses and may contribute to atherosclerosis (41). Immunologic arterial injury due to allograft rejection acting in synergy with hypercholesterolemia resulting from a dietary supplement of cholesterol can lead to rapidly developing atherosclerosis (42). Autoimmune thyroid disease has a causal relationship with atherosclerosis (even if mediated through traditional risk factors) (43). Focal adhesion plays key roles in VSMCs. Focal adhesion pathways may be expected to facilitate the formation of atherosclerotic plaques in ApoE-null mice (44). Systemic lupus erythematosus (SLE) is a systemic autoimmune disease that is characterized by autoantibody production and inflammatory disease involving multiple organs. Premature atherosclerosis is a common complication of SLE and results in substantial morbidity and mortality from cardiovascular disease (CVD) (45). Diabetes and atherosclerosis are associated with disorders of lipids and lipoproteins, notably high apolipoprotein B (apoB) and low apolipoprotein A1 (apoA1) are well established (46). Type I diabetes mellitus was also enriched by differential genes in this study. The passage of leukocytes across the endothelium and into arterial walls is a critical step in the development of atherosclerosis. It is consistent with our observation that DEGs were enriched in the pathway 'leukocyte transendothelial migration' (47).

The expression levels of matrix metallopeptidase 9 (MMP9) were assessed. MMP9 was potentially important in the development of atherosclerosis in a Malaysian study population (13). Cystic fibrosis transmembrane conductance regulator (CFTR) has a similar function to ABCA1. Schmitz and Buechler (48) identified ABCA1 as the major regulator of plasma high density lipoprotein (HDL) cholesterol. HDL metabolism is crucial in the prevention of the progression of atherosclerosis. CFL2 is an interactor of CFTR as reported by Wang and colleagues (49). An in vivo ApoE $\mathrm{Ap}^{-/-}$mouse model was utilized to assess the effects of chronic moderate exposure to arsenic on plaque formation and composition in order to facilitate mechanistic investigations (50). Arsenic exposure increases oxidative stress, inflammation and atherosclerotic lesion formation in $\mathrm{ApoE}^{-/-}$mice (51). In human arterial tissue, SERPINA1 was upregulated (6.3-fold) in atherosclerotic plaques (14). In the present study, SERPINA1 was also upregulated in the advanced stage.

In this study, we also found that miR-328 may be crucial for atherosclerosis. miR-328 was linked to multiple upregulated genes in advanced stage samples and miR-328 has been found to be antiangiogenic (52). Anti-angiogenic perfluorocarbon nanoparticles has already used for diagnosis and treatment of atherosclerosis (53). Recently, in the ABCG2-positive cancer cells, miR-328 has been reported to regulate the expression of BCRP/ABCG2 (54). Additionally, miR-328 expression in plasma was significantly increased in atrial fibrillation (AF) patients (55).

Motifs enriched by upregulated genes in the early and advanced stages including Myf, MZF1, FOXL1 and NKX3-1, which were not investigated extensively were also investigated. These transcriptional factors may play pivotal roles in carotid atherosclerosis. In the present study, we identified gene expression changes and regulatory factors in carotid atherosclerosis.
These results may facilitate in identifying the mechanism involved in carotid atherosclerosis.

\section{References}

1. Ross R: The pathogenesis of atherosclerosis: a perspective for the 1990s. Nature 362: 801-809, 1993.

2. Maseri A and Fuster V: Is there a vulnerable plaque? Circulation 107: 2068-2071, 2003.

3. Ross R: Atherosclerosis - an inflammatory disease. N Engl J Med 340: 115-126, 1999.

4. O'Leary DH, Polak JF, Kronmal RA, Manolio TA, Burke GL and Wolfson SK Jr: Carotid-artery intima and media thickness as a risk factor for myocardial infarction and stroke in older adults. Cardiovascular Health Study Collaborative Research Group. N Engl J Med 340: 14-22, 1999.

5. Wu Y, Tao Z, Song C, et al: Overexpression of YKL-40 predicts plaque instability in carotid atherosclerosis with CagA-positive helicobacter pylori infection. PLoS One 8: e59996, 2013.

6. Yamada M, Kim S, Egashira K, et al: Molecular mechanism and role of endothelial monocyte chemoattractant protein-1 induction by vascular endothelial growth factor. Arterioscler Thromb Vasc Biol 23: 1996-2001, 2003.

7. Leng XY, Chen XY, Chook P, et al: Association between metabolic syndrome and carotid atherosclerosis: a communitybased study in Hong Kong. Metab Syndr Relat Disord 11: 109-114, 2013.

8. Ota H, Reeves MJ, Zhu DC, et al: Sex differences of high-risk carotid atherosclerotic plaque with less than $50 \%$ stenosis in asymptomatic patients: an in vivo 3T MRI study. AJNR Am J Neuroradiol 34: 1049-1055, 2013.

9. Ino-Oka E, Sekino H, Kajikawa S, Satoh T and Inooka H: Evaluation of carotid atherosclerosis from the perspective of blood flow reflection. Clin Exp Hypertens 31: 188-200, 2009.

10. Maeda S, Sawayama Y, Furusyo N, Shigematsu M and Hayashi J: The association between fatal vascular events and risk factors for carotid atherosclerosis in patients on maintenance hemodialysis: plaque number of dialytic atherosclerosis study. Atherosclerosis 204: 549-555, 2009.

11. Munakata M, Sakuraba J, Tayama J, et al: Higher brachial-ankle pulse wave velocity is associated with more advanced carotid atherosclerosis in end-stage renal disease. Hypertens Res 28: 9-14, 2005.

12. Hashimoto H, Kitagawa K, Kuwabara K, et al: Circulating adhesion molecules are correlated with ultrasonic assessment of carotid plaques. Clin Sci (Lond) 104: 521-527, 2003.

13. Blin J, Ahmad Z, Rampal LR, Mohtarrudin N, Tajudin AK and Adnan RS: Preliminary assessment of differential expression of candidate genes associated with atherosclerosis. Genes Genet Syst 88: 199-209, 2013.

14. Inouye M, Ripatti S, Kettunen J, et al: Novel Loci for metabolic networks and multi-tissue expression studies reveal genes for atherosclerosis. PLoS Genet 8: e1002907, 2012.

15. Bateman HR, Liang Q, Fan D, Rodriguez V and Lessner SM: Sparstolonin B inhibits pro-angiogenic functions and blocks cell cycle progression in endothelial cells. PLoS One 8: e70500, 2013.

16. Feig JE, Vengrenyuk Y, Reiser V, et al: Regression of atherosclerosis is characterized by broad changes in the plaque macrophage transcriptome. PLoS One 7: e39790, 2012.

17. Zhang E and Wu Y: Dual effects of miR-155 on macrophages at different stages of atherosclerosis: LDL is the key? Med Hypotheses 2014: 83:74-78, 2014.

18. Bonaterra GA, Zugel S, Thogersen J, et al: Growth differentiation factor-15 deficiency inhibits atherosclerosis progression by regulating interleukin-6-dependent inflammatory response to vascular injury. J Am Heart Assoc 1: e002550, 2012.

19. Orr AW, Hastings NE, Blackman BR and Wamhoff BR: Complex regulation and function of the inflammatory smooth muscle cell phenotype in atherosclerosis. J Vasc Res 47: 168-180, 2010.

20. States JC, Singh AV, Knudsen TB, et al: Prenatal arsenic exposure alters gene expression in the adult liver to a proinflammatory state contributing to accelerated atherosclerosis. PLoS One 7: e38713, 2012.

21. Bahls M, Bidwell CA, Hu J, et al: Gene expression differences during the heterogeneous progression of peripheral atherosclerosis in familial hypercholesterolemic swine. BMC Genomics 14: 443, 2013. 
22. Pinkaew D, Hutadilok-Towatana N, Teng BB, Mahabusarakam W and Fujise K: Morelloflavone, a biflavonoid inhibitor of migration-related kinases, ameliorates atherosclerosis in mice. Am J Physiol Heart Circ Physiol 302: H451-H458, 2012.

23. Doring Y, Manthey HD, Drechsler M, et al: Auto-antigenic protein-DNA complexes stimulate plasmacytoid dendritic cells to promote atherosclerosis. Circulation 125: 1673-1683, 2012.

24. Irizarry RA, Hobbs B, Collin F, et al: Exploration, normalization, and summaries of high density oligonucleotide array probe level data. Biostatistics 4: 249-264, 2003.

25. Philip R: Semantic similarity in a taxonomy: an informationbased measure and its application to problems of ambiguity in natural language. J Artif Intell Res 11: 95-130, 1999.

26. Huang da W, Sherman BT and Lempicki RA: Systematic and integrative analysis of large gene lists using DAVID bioinformatics resources. Nat Protoc 4: 44-57, 2009.

27. Portales-Casamar E, Thongjuea S, Kwon AT, et al: JASPAR 2010: the greatly expanded open-access database of transcription factor binding profiles. Nucleic Acids Res 38: D105-D110, 2010.

28. Wang $\mathrm{K}, \mathrm{Hu} \mathrm{F}, \mathrm{Xu} \mathrm{K}$, et al: CASCADE_SCAN: mining signal transduction network from high-throughput data based on steepest descent method. BMC Bioinformatics 12: 164, 2011.

29. Kruger J and Rehmsmeier M: RNAhybrid: microRNA target prediction easy, fast and flexible. Nucleic Acids Res 34: W451-W454, 2006.

30. Li LX, Zhao CC, Ren Y, et al: Prevalence and clinical characteristics of carotid atherosclerosis in newly diagnosed patients with ketosis-onset diabetes: a cross-sectional study. Cardiovasc Diabetol 12: 18, 2013.

31. Li X, Ni R, Chen J, et al: The presence of IGHG1 in human pancreatic carcinomas is associated with immune evasion mechanisms. Pancreas 40: 753-761, 2011.

32. Pan B, Zheng S, Liu C and Xu Y: Suppression of IGHG1 gene expression by siRNA leads to growth inhibition and apoptosis induction in human prostate cancer cell. Mol Biol Rep 40: 27-33, 2013.

33. Kitaya K, Yasuo T, Yamaguchi T, Fushiki S and Honjo H: Genes regulated by interferon- $\gamma$ in human uterine microvascular endothelial cells. Int J Mol Med 20: 689-697, 2007.

34. Shan M, Yuan X, Song LZ, et al: Cigarette smoke induction of osteopontin (SPP1) mediates $\mathrm{T}_{\mathrm{H}} 17$ inflammation in human and experimental emphysema. Sci Transl Med 4: 117ra9, 2012.

35. Lohr M, Edlund K, Botling J, et al: The prognostic relevance of tumour-infiltrating plasma cells and immunoglobulin kappa $\mathrm{C}$ indicates an important role of the humoral immune response in non-small cell lung cancer. Cancer Lett 333: 222-228, 2013.

36. Whiteside TL and Ferrone S: IGKC and prognosis in breast cancer - response to Schmidt. Clin Cancer Res 19: 305, 2013.

37. Schmidt M, Micke P and Hengstler JG: IGKC and prognosis in breast cancer. Clin Cancer Res 19: 304, 2013.

38. Luchtefeld M, Preuss C, Ruhle F, et al: Gp130-dependent release of acute phase proteins is linked to the activation of innate immune signaling pathways. PLoS One 6: e19427, 2011.

39. Hahn C and Schwartz MA: Mechanotransduction in vascular physiology and atherogenesis. Nat Rev Mol Cell Biol 10: 53-62, 2009.

40. Sjoland H, Eitzman DT, Gordon D, Westrick R, Nabel EG and Ginsburg D: Atherosclerosis progression in LDL receptordeficient and apolipoprotein E-deficient mice is independent of genetic alterations in plasminogen activator inhibitor-1. Arterioscler Thromb Vasc Biol 20: 846-852, 2000.
41. Languino LR, Duperray A, Joganic KJ, Fornaro M, Thornton GB and Altieri DC: Regulation of leukocyte-endothelium interaction and leukocyte transendothelial migration by intercellular adhesion molecule 1-fibrinogen recognition. Proc Natl Acad Sci USA 92: 1505-1509, 1995.

42. Alonso DR, Starek PK and Minick CR: Studies on the pathogenesis of atheroarteriosclerosis induced in rabbit cardiac allografts by the synergy of graft rejection and hypercholesterolemia. Am J Pathol 87: 415-442, 1977.

43. McLeod DS: Autoimmune thyroid disease: a novel risk factor for atherosclerosis? Endocrine 44: 8-10, 2013.

44. Bu DX, Rai V, Shen X, et al: Activation of the ROCK1 branch of the transforming growth factor- $\beta$ pathway contributes to RAGEdependent acceleration of atherosclerosis in diabetic ApoE-null mice. Circ Res 106: 1040-1051, 2010.

45. Richez C, Richards RJ, Duffau P, et al: The effect of mycophenolate mofetil on disease development in the gld.apo $E^{-r}$ mouse model of accelerated atherosclerosis and systemic lupus erythematosus. PLoS One 8: e61042, 2013.

46. Hashemi M, Saadat M, Behjati M and Kelishadi R: Comparison of serum apolipoprotein levels of diabetic children and healthy children with or without diabetic parents. Cholesterol 2012: 490381, 2012.

47. Samson T, van Buul JD, Kroon J, et al: The guanine-nucleotide exchange factor SGEF plays a crucial role in the formation of atherosclerosis. PLoS One 8: e55202, 2013.

48. Schmitz G and Buechler C: ABCA1: regulation, trafficking and association with heteromeric proteins. Ann Med 34: 334-347, 2002.

49. Wang X, Venable J, LaPointe P, et al: Hsp90 cochaperone Aha1 downregulation rescues misfolding of CFTR in cystic fibrosis. Cell 127: 803-815, 2006.

50. Lemaire M, Lemarie CA, Molina MF, Schiffrin EL, Lehoux S and Mann KK: Exposure to moderate arsenic concentrations increases atherosclerosis in $\mathrm{ApoE}^{-/-}$mouse model. Toxicol Sci 122: 211-221, 2011.

51. Srivastava S, Vladykovskaya EN, Haberzettl P, Sithu SD, D'Souza SE and States JC: Arsenic exacerbates atherosclerotic lesion formation and inflammation in ApoE-/- mice. Toxicol Appl Pharmacol 241: 90-100, 2009.

52. Suarez Y and Sessa WC: MicroRNAs as novel regulators of angiogenesis. Circ Res 104: 442-454, 2009.

53. Caruthers SD, Cyrus T, Winter PM, Wickline SA and Lanza GM: Anti-angiogenic perfluorocarbon nanoparticles for diagnosis and treatment of atherosclerosis. Wiley Interdiscip Rev Nanomed Nanobiotechnol 1: 311-323, 2009.

54. Li X, Pan YZ, Seigel GM, Hu ZH, Huang M and Yu AM: Breast cancer resistance protein $\mathrm{BCRP} / \mathrm{ABCG} 2$ regulatory microRNAs (hsa-miR-328, -519c and -520h) and their differential expression in stem-like $\mathrm{ABCG}^{+}$cancer cells. Biochem Pharmacol 81: 783-792, 2011.

55. Wang X, Qiu CG, Huang ZW, Han ZY, Lu WJ and Chen XJ: The expression and clinical implication of plasma miR-328 in patients with atrial fibrillation. Zhonghua Xin Xue Guan Bing Za Zhi 41: 126-129, 2013 (In Chinese). 